\title{
Anion charge-lattice volume maps for searching lithium superionic conductors
}

\author{
Zhenming $\mathrm{Xu}^{\mathrm{a}, \mathrm{b}}$, Hong $\mathrm{Zhu}^{\mathrm{a}}{ }^{*}$ \\ a. University of Michigan-Shanghai Jiao Tong University Joint Institute, Shanghai Jiao Tong \\ University, 800 Dongchuan Road, Shanghai, 200240, China \\ b. John. A. Paulson School of Engineering and Applied Sciences, Harvard University, 29 \\ Oxford Street, Cambridge, Massachusetts, 02138, USA
}

\begin{abstract}
The effects of anion charge and lattice volume (lithium-anion bond length) on lithium ion migration have been investigated by utilizing the density functional theory calculations combined with the anion framework models, e.g. $f c c, h c p$ and $b c c$. It is found that the anion charge and lattice volume have great impacts on the activation energy barrier $\left(E_{a}\right)$ of lithium ion migration, which is validated by some reported sulfides. For the tetrahedrally occupied lithium, the less negative anion charge is, the lower the lithium ion migration barrier is likely to be. While for the octahedrally occupied lithium, the more negative anion charge is, the lower the lithium ion migration barrier is. The large lattice volume (lithium-anion bond length) can lower $E_{a}$ to a certain extent. Lithium ion direct migrations along the direct Tet-Tet pathway in the bcc-or hcp-type anion framework are less sensitive to anion charge and lattice volume than other pathways. Most importantly, based on the full understandings of anion framework model, new design rules for developing alkali-metal superionic conductors were proposed. Getting the desired electronegativity difference between the anion element and non-mobile cation element by selecting the most suitable non-mobile cation element without changing the crystal structure framework can eventually achieve low $\mathrm{E}_{\mathrm{a}}$ for alkali-metal ion migration. For the desired alkali-metal superionic conductors with tetrahedrally occupied alkali-metal ions, the fine non-mobile cation element should give preferences to those elements located at the right top of the periodic table of elements with large electronegativities. For the alkali-metal superionic conductors with octahedrally occupied alkali-metal ions, the fine non-mobile cation element should give preferences to the elements located at the left bottom of the periodic table with small electronegativities.
\end{abstract}

${ }^{*}$ Corresponding author:

E-mail: hong.zhu@sjtu.edu.cn 
Keywords: ionic conductor; lithium ion migration; anion framework; anion charge; lattice volume; design rule

\section{Introduction}

Today, lithium ion battery (LIB) techniques provide much conveniences for people's life, powering the portable electronic devices ${ }^{1}$. However, we encounter the safety issues when using the commercial LIBs in the application scenarios of the large-scale energy storage in electric vehicles, because of the flammability of liquid organic electrolyte ${ }^{2-3}$. Fortunately, replacing the currently employed flammable organic electrolytes with the inflammable solid-state electrolyte (SSE) materials and matching with the lithium metal anodes to construct the all-solid-state lithium ion batteries (ASSLIBs) not only solve the safety issues, but also drastically promote the energy density of $\mathrm{LIBs}^{4-6}$, making electric cars run farther. Correspondingly, high room temperature lithium ionic conductivities $\left(10^{-3}-10^{-2} \mathrm{~S} \mathrm{~cm}^{-1}\right)$ of SSE materials are the essential prerequisite for constructing ASSLIB systems. At present, some lithium superionic conductors, e.g. $\mathrm{Li}_{7} \mathrm{La}_{3} \mathrm{Zr}_{2} \mathrm{O}_{12}{ }^{7}, \mathrm{LiTi}_{2}\left(\mathrm{PO}_{4}\right)_{3}{ }^{8}$ oxides and $\mathrm{Li}_{10} \mathrm{GeP}_{2} \mathrm{~S}_{12}$ (LGPS) ${ }^{9}, \mathrm{Li}_{7} \mathrm{P}_{3} \mathrm{~S}_{11}{ }^{10}$ sulfides, have been widely studied and successfully developed as the SSE materials, and the state-of-the-art room temperature ionic conductivities of $12-17 \mathrm{mS} \mathrm{cm} \mathrm{cm}^{-1}$ are experimentally realized in $\mathrm{Li}_{10} \mathrm{GeP}_{2} \mathrm{~S}_{12}$ and $\mathrm{Li}_{7} \mathrm{P}_{3} \mathrm{~S}_{11}$ sulfides. To efficiently exploit more advanced lithium superionic conductors, it's quite necessary to deeply understand the fast ion migration mechanism in the state-of-the-art superionic conductors, and even propose proper design rules.

Among the various studies on understanding fast ion migration, the topology analysis of lithium migration pathways is possibly the most common and useful method ${ }^{11-13}$. Ceder et al. calculated the topology features of the anion framework structures of various lithium sulfides, revealed a fundamental relationship between anion packing pattern and lithium ionic transport, and eventually proposed the design concept of body-centered cubic-like anion framework with face-shared tetrahedral lithium sites for fast ion migration ${ }^{11}$. Subsequently, they utilized this design concept and computationally predicted a new lithium superionic conductor, $\mathrm{Li}_{1+2 x} \mathrm{Zn}_{1-\mathrm{x}} \mathrm{PS}_{4}$, with exceptionally high lithium ion conductivity at room temperature ${ }^{14}$. In addition, Mo et al. pointed out the unique feature of the common lithium superionic conductors with abundant enlarged lithium sites caused by large local spaces (fractionally occupied lithium sites) in the crystal structural framework, and identified many new structures as fast lithium-ion conductors by the high-throughput screening 
combined with this quantified feature ${ }^{13}$. However, these understandings based on the topology of lithium migration channels alone are insufficient to explain the enhanced ionic conductivity by the $\mathrm{Cl}$ doping and substitution in lithium sulfides. The $\mathrm{Cl}$-doped $\mathrm{Li} 9.54 \mathrm{Si}_{1.74} \mathrm{P}_{1.44} \mathrm{~S}_{11.7} \mathrm{Cl}_{0.3}{ }^{15}$ shows a quite high ionic conductivity of $25 \mathrm{mS} / \mathrm{cm}$ at room temperature (twice that of the original $\mathrm{LGPS}^{9}$ ), in which thoroughly substituting Ge in LGPS with Si and partially replacing S with $\mathrm{Cl}$ have negligible influence on the lattice constants, the local atom arrangements and even the morphologies of lithium ion migration channel ${ }^{15}$. In addition, $\mathrm{Li}_{5.5} \mathrm{PS}_{4.5} \mathrm{Cl}_{1.5}$ with more substituted $\mathrm{Cl}$ shows higher room temperature ionic conductivities of $9.4 \mathrm{mS} / \mathrm{cm}$ (cold-pressed) and $12 \mathrm{mS} / \mathrm{cm}$ (sintered) ${ }^{16}$ than the normal $\mathrm{Li}_{6} \mathrm{PS}_{5} \mathrm{Cl}$ of $2.4 \mathrm{mS} / \mathrm{cm}^{17}$, in spite of the negative factors of reduced lattice constant $(9.81 \AA$ vs. $9.86 \AA)$ and lower lithium concentration resulted from the extensive $\mathrm{Cl}$ substitution. Thus, there would be other important factors influencing the lithium ionic conductivity in addition to the topology-based structure features. As we know, in ionic materials, the Columbic interaction dominates the interaction between lithium cation and its adjacent anion framework ${ }^{18-21}$ and the monovalent halogen anions have weaker interactions with lithium ions than divalent sulfur or oxygen anions ${ }^{22}$. Yet, as far as we know, there is no systematical understanding of the Columbic interaction dependent lithium ion migration, which remains to be uncovered as regards this issue. Therefore, this study aims to fill the existing knowledge gaps regarding the effect of Columbic interaction on lithium ion migration in solids.

In the most crystalline lithium compounds, lithium ions occupy the tetrahedral (Tet) or octahedral $(O c t)$ sites, forming the stable tetrahedral or octahedral lithium-anion polyhedrons, and the anion packing modes could be approximately classified into the face-centered cubic $(f c c)$, body-centered cubic (bcc) and hexagonal close-packed ( $h c p$ ) frameworks ${ }^{11,23}$. The anion frameworks of $\mathrm{LiCoO}_{2}$, $\mathrm{Li}_{2} \mathrm{MnO}_{3}, \mathrm{Li}_{4} \mathrm{Ti}_{5} \mathrm{O}_{12}, \mathrm{Li}_{2} \mathrm{~S}, \mathrm{LiTiS}_{2}$ and $\mathrm{Li}_{3} \mathrm{YBr}_{6}{ }^{22}$ can be exactly matched to the $f c c$ types. In $\mathrm{Li}_{7} \mathrm{P}_{3} \mathrm{~S}_{11}$ and $\mathrm{Li}_{10} \mathrm{GeP}_{2} \mathrm{~S}_{12}$, the sulfur anion frameworks can be roughly mapped to the $b c c$ lattices with some distortions. In $\gamma$ - $\mathrm{Li}_{3} \mathrm{PS}_{4}$ and $\mathrm{Li}_{4} \mathrm{GeS}_{4}$, the $\mathrm{S}$ anion frameworks can be closely matched to the $h c p$ arrays ${ }^{11}$. Our previous studies of the $f c c$ anion frameworks show that the anion charge and anion volume have great impacts on the stability of lithium ion occupation and lithium ion migration ${ }^{19,21,}$ 23-24. Considering the excellent lithium ion migration usually occurring in the sulfide-type superionic conductors with the $b c c$ and $h c p$ sulfur anion frameworks, such as $\mathrm{Li}_{7} \mathrm{P}_{3} \mathrm{~S}_{11}, \mathrm{Li}_{10} \mathrm{GeP}_{2} \mathrm{~S}_{12}$, and $\gamma$ - $\mathrm{Li}_{3} \mathrm{PS}_{4}$, in this work, we would shed light on the effects of the anion charge and lattice volume dependent Coulomb interaction on the lithium ion migration in the $b c c$ and $h c p$ anion frameworks. Moreover, the anion charge-lattice volume maps were depicted and new design rules were proposed for efficiently searching the advanced alkali-metal superionic conductors. 


\section{Computational methodologies}

All calculations were performed by using the Vienna $a b$-initio Simulation Package (VASP) software based on the projector augmented wave (PAW) method in the framework of the density functional theory $(\mathrm{DFT})^{25}$. The Perdew-Burke-Ernzerhof (PBE) exchange functional ${ }^{26}$ in the framework of generalized gradient approximation (GGA) ${ }^{27}$ was utilized to solve the Schrödinger's equation of the quantum states of electrons. The energy cutoff of plane-wave is $500 \mathrm{eV}$. The convergence criteria of energy and force are $10^{-5} \mathrm{eV} /$ atom and $0.01 \mathrm{eV} / \AA$, respectively. The Monkhorst-Pack method ${ }^{28}$ with $4 \times 4 \times 4$ and $4 \times 4 \times 2 \quad k$-point mesh were employed for the Brillouin zone sampling of the body-centered cubic and hexagonal close-packed anion sublattices, respectively. The energy landscapes and activation energy barrier of lithium ion migration in the anion frameworks are calculated by the nudged elastic band (NEB) method ${ }^{29}$. During the NEB calculations, only one lithium ion is allowed to move, while the sulfur(oxygen) anions are fixed in their initial positions, and this approach has been also utilized by Ceder et $\mathrm{al}^{11}$. The anion charges in the anion framework are changed by adjusting the uniform background charges of the whole framework system.

\section{Results and discussion}

\subsection{Anion charge and lattice volume dependent lithium occupation and migration}

First, to get the reasonable ranges of anion charge and lattice volume for the following NEB calculations, the scatter distributions of the anion Bader charges and lattice volumes of lithium oxides and sulfides were investigated and shown in Figure S1, which were obtained from the AFLOW database without any DFT calculation ${ }^{30}$. It can be found that the oxygen anion charges are mainly spread in the range of $[-0.8 e,-1.7 e]$, while the sulfur anion charges are principally distributed in the range of [-0.7 e, -1.5e]. Thereby, a wider anion charge range of $[-0.5 e,-1.8 e]$, simultaneously covering the dominating oxygen and sulfur anion charges, was considered for the following NEB calculations. The lattice volumes of the oxides and sulfides are mainly spread in the 
ranges of [15 $\AA^{3}$ /atom, $30 \AA^{3} /$ atom] and [30 $\AA^{3} /$ atom, $50 \AA^{3} /$ atom], respectively. In addition, the Bader charges of lithium cation in the lithium oxides and sulfides are dominantly located in the range of $[+0.84 e \sim+0.92 e]$ (Figure $\mathrm{S} 2$ ), which are far narrower than those of anions. It indicates that the charges of lithium ion in different compounds don't change a lot, and thereby can be regarded as a constant.

Then, two artificial $b c c$ - and $h c p$-type anion frameworks with a single lithium cation and 16 anions were built to investigate lithium ion migration in them, as illustrated in Figure 1. Only two chalcogen anion elements (oxygen and sulfur) were considered for the anion framework models in view of the fact that the most reported lithium superionic conductors are lithium oxides and sulfides. These sulfur(oxygen) anion framework models can allow us directly capture the effects of the Coulomb interactions between lithium cation and its adjacent anion, which fundamentally depend on the anion charge and lattice volume. Note that the Coulomb interactions between lithium cation and its adjacent anion fundamentally depend on the anion charge and lithium-anion bond length. In this work, for the convenience of the NEB energy landscape calculations, we varied the anion sublattice volume (averaged to per anion atom, unit cell volume/number of anion) instead of the lithium-anion bond length, because there is a linear positive relationship between the lattice volume and lithium-anion bond length in the anion framework model. There is only one kind of tetrahedral interstitial site in the $b c c$-type anion framework (Figure 1a), corresponding to a Tet-Tet lithium ion migration pathway, and this direct lithium ion hopping between two adjacent Tet sites has been reported to possess the lowest activation barrier and the highest lithium ionic conductivity ${ }^{11}$. For the $h c p$-type anion framework model, there are two different kinds of interstitial sites, including the tetrahedral and octahedral interstitial sites (Figure 1b). Thus, the occupied lithium ion in the $h c p$ anion framework could migrate along three different direct pathways, including Tet-Tet, Oct-Oct and Oct-Tet pathways, and the latter is the half of the Tet-Oct-Tet and Oct-Tet-Oct pathways. 
(a)

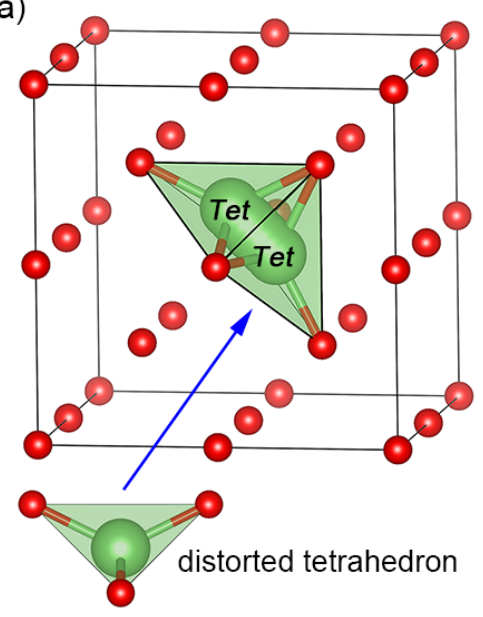

(b)

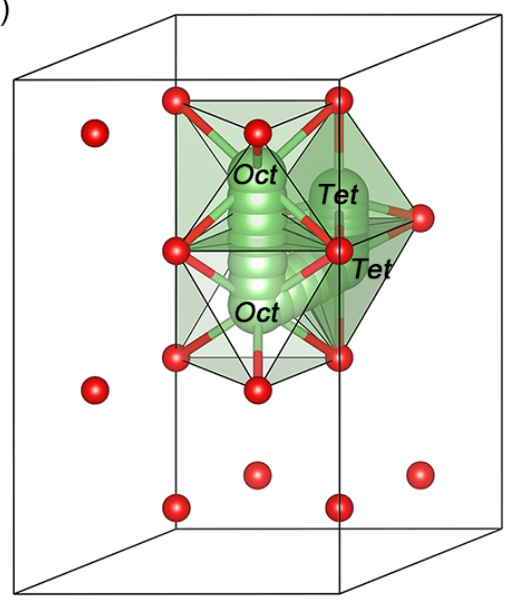

Figure 1. Structural models of (a) lithium ion migration along the Tet-Tet pathway (two adjacent tetrahedral sites) in the $b c c$-type anion framework with a lithium cation and 16 anions, the lithium-anion tetrahedrons in the bcc-type anion framework are distorted with a csm (continuous symmetry measure) value of 2.29 (a csm of 0 corresponds to a perfect tetrahedron); (b) lithium ion migration along the Tet-Tet pathway, Oct-Oct pathway (octahedral site to octahedral site), and Oct-Tet pathway in the hcp-type anion framework with a lithium cation and 16 anions. The anions are colored red, and lithium ions are colored green, respectively.

\subsection{1 bcc-type anion frameworks}

Then, we performed NEB calculations to get the energy landscapes of lithium ion migration in the $b c c$-type sulfur(oxygen) anion frameworks with respect to different anion charges and lattice volumes. Lithium ion migration between two face-shared distorted lithium-anion tetrahedral sites (namely Tet-Tet pathway) in the bcc-type anion framework passes through a distorted triangular anion bottleneck (Figure 1a), whose relative energy with respect to the Tet site corresponds to the activation energy barrier $\left(E_{a}\right)$ of lithium ion migration. The $E_{a}$ of lithium ion migration in the $b c c$-type sulfur(oxygen) anion frameworks are shown in Figure 2 and S3, respectively. To clearly show the $E_{a}$ variations with respect to different anion charges at any constant lattice volume, the heatmaps of Figure 2 and S3 are transformed into the facetgrid plots, as illustrated in Figure S5 and S6, respectively. It can be clearly seen that the anion charge and lattice volume show the similar influences on $\mathrm{E}_{\mathrm{a}}$ for both sulfur and oxygen anion frameworks, but the maximum of $\mathrm{E}_{\mathrm{a}}$ for the sulfur anion framework is smaller than that of the oxygen anion framework system, due to the better polarizability of sulfur anion. The $\mathrm{E}_{\mathrm{a}}$ variation ranging from 0 to $0.42 \mathrm{eV}$ in the whole anion charge and lattice volume space of the bcc-type oxygen anion framework (Figure S3) is much narrower than that of the $f c c$-type oxygen anion framework from 0.25 to $1.5 \mathrm{eV}$ (Figure S4a). In addition, the maximum variations of $E_{a}$ with respect to different anion charges at any constant lattice volume are 
$\sim 0.12$ and $0.07 \mathrm{eV}$, respectively for the oxygen and sulfur anion framework (Figure S5 and S6). They indicate that $\mathrm{E}_{\mathrm{a}}$ of lithium ion migration in the $b c c$-type anion frameworks are not only smaller but also less sensitive to the variations of anion charge than that in the $f c c$-type anion framework. It's worth noting that the lithium compounds possessing the $b c c$-type anion frameworks are much fewer than those with the $f c c$ - and $h c p$-anion frameworks (Table $\mathrm{S} 1)^{23}$, although they usually show higher lithium ion conductivities ${ }^{11}$. Overall, the anion charge and lattice volume (lithium-anion bond length) dependent Coulomb interactions have smaller impacts on $\mathrm{E}_{\mathrm{a}}$ of lithium ion migration along the Tet-Tet pathways in the $b c c$-type anion frameworks.

In the whole anion charge and lattice volume space, the more negative anion charges can lead to slightly higher $\mathrm{E}_{\mathrm{a}}$ for lithium ion migration in the bcc-type anion framework along the Tet-Tet pathways at any constant lattice volume (Figure S5 and S6), and the large lattice volumes can reduce $E_{a}$ at any constant anion charge (Figure 2 and S3). In short, the little negative anion charges and large lattice volumes can lead to lower $\mathrm{E}_{\mathrm{a}}$. Therefore, the little negative anion charges and large lattice volumes (lithium-anion bond lengths) are essential for achieving fast lithium ion migration in lithium compounds with the $b c c$-type anion frameworks. It's worth noting that these variation trends of $\mathrm{E}_{\mathrm{a}}$ with respect to anion charge and lattice volume are much more interesting than the absolute values of $E_{a}$. These observed trends are also found in the previous experimental results for some sulfide-type lithium conductors with the $b c c$-type anion frameworks. The higher element electronegativity of $\mathrm{Ge}^{4+}$ vs. $\mathrm{Sn}^{4+}(2.116 \text { vs. 1.877) })^{31}$ lead to less negative sulfur anion charges in $\mathrm{Li}_{10} \mathrm{GeP}_{2} \mathrm{~S}_{12}$ (-0.151e vs. -0.168 e in $\mathrm{Li}_{10} \mathrm{SnP}_{2} \mathrm{~S}_{12}$, Hirshfeld charge $)^{21}$, and the Li-S average bond length of $\mathrm{Li}_{10} \mathrm{GeP}_{2} \mathrm{~S}_{12}$ is $2.585 \AA$, slightly larger than $2.578 \AA$ of $\mathrm{Li}_{10} \mathrm{SnP}_{2} \mathrm{~S}_{12}{ }^{21}$. The relatively less negative sulfur anion charge and larger Li-S average bond length eventually lead to relatively lower $\mathrm{E}_{\mathrm{a}}$ of $\mathrm{Li}_{10} \mathrm{GeP}_{2} \mathrm{~S}_{12}$ compared to $\mathrm{Li}_{10} \mathrm{SnP}_{2} \mathrm{~S}_{12}{ }^{32}$, which is well consistent with the predicted theoretical trends from the $a b$-initio molecular dynamics (AIMD) simulations by S. P. Ong et $\mathrm{al}^{12}$. The enhanced ionic conductivities can also be obtained by the monovalent $\mathrm{Cl}$ element doping in lithium sulfides, the $\mathrm{Cl}$-doped $\mathrm{Li}{ }_{9.54} \mathrm{Si}_{1.74} \mathrm{P}_{1.44} \mathrm{~S}_{11.7} \mathrm{Cl}_{0.3}{ }^{15}$ shows a quite higher ionic conductivity of 25 $\mathrm{mS} / \mathrm{cm}$ at room temperature than LGPS $^{9}$, in which thoroughly substituting Ge of LGPS with Si and partially replacing $\mathrm{S}$ with $\mathrm{Cl}$ has negligible influence on the lattice constants and even the morphologies of lithium ion migration channel ${ }^{15}$. Furthermore, a recent DFT research shows that doping a small number of oxygen atoms into $\mathrm{Li}_{7} \mathrm{P}_{3} \mathrm{~S}_{11}$ with the $b c c$-type sulfur anion framework can significantly reduce $\mathrm{E}_{\mathrm{a}}$ of lithium ion migration, and enhance lithium ion conductivity from $50 \mathrm{mS}$ $\mathrm{cm}^{-1}$ of the pristine $\mathrm{Li}_{7} \mathrm{P}_{3} \mathrm{~S}_{11}$ to $109 \mathrm{mS} \mathrm{cm}{ }^{-1}$ of the oxygen-doped $\mathrm{Li}_{7} \mathrm{P}_{3} \mathrm{~S}_{10.25} \mathrm{O}_{0.75}$ at $300 \mathrm{~K}$, in which the Bader charge of $\mathrm{S}$ atom changes from $-0.89 e$ (pristine) to $-0.88 e$ (oxygen-doped) ${ }^{33}$. These 
consistent variation tendencies of $\mathrm{E}_{\mathrm{a}}$ in the cation-substituted $\mathrm{Li}_{10} \mathrm{MP}_{2} \mathrm{~S}_{12}(\mathrm{M}=\mathrm{Ge}$ and $\mathrm{Sn})$, chlorine-doped $\mathrm{Li}_{9.54} \mathrm{Si}_{1.74} \mathrm{P}_{1.44} \mathrm{~S}_{11.7} \mathrm{Cl}_{0.3}$ and oxygen-doped $\mathrm{Li}_{7} \mathrm{P}_{3} \mathrm{~S}_{10.25} \mathrm{O}_{0.75}$ materials are fully consistent with our findings, validating the reasonability of the $b c c$-type anion framework model and the corresponding analyses of Coulomb interactions.

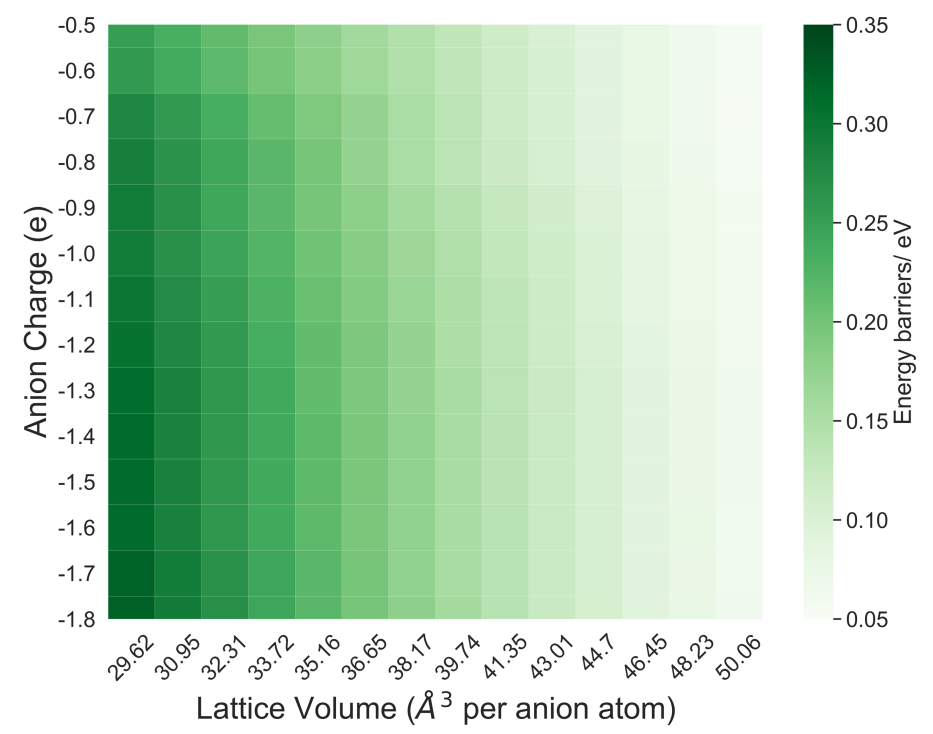

Figure 2. Heat maps of the calculated $\mathrm{E}_{\mathrm{a}}$ of lithium ion migration in the $b c c$-type sulfur anion frameworks along the Tet-Tet migration pathway with respect to anion charge and lattice volume.

\subsection{2 hcp-type anion frameworks}

Different from the less common $b c c$-type anion frameworks ${ }^{23}$, there are both tetrahedral and octahedral interstitial sites in the $h c p$-type anion framework model (Figure 1b). Therefore, the discussions about the lithium ion migration in the $h c p$-type anion framework are organized based on three different pathways, including Tet-Tet, Oct-Oct and Oct-Tet.

\subsubsection{Tet-Tet pathway}

The calculated $\mathrm{E}_{\mathrm{a}}$ of lithium ion migration in the hcp-type sulfur(oxygen) anion frameworks along the direct Tet-Tet pathways with respect to anion charge and lattice volume are shown in Figure 3a, $\mathrm{S} 7, \mathrm{~S} 8$ and S9. It can be observed that $\mathrm{E}_{\mathrm{a}}$ varies from 0 to $0.5 \mathrm{eV}$ in the whole anion charge and lattice volume space for the Tet-Tet migration pathways in the hcp-type sulfur(oxygen) anion framework (Figure 3a and S7), whose ranges are similar to that of the Tet-Tet migration in the 
bcc-type sulfur(oxygen) anion frameworks (Figure 2 and S3) $(0-0.42 \mathrm{eV})$, and narrower than that of the $f c c$-type oxygen anion framework from 0.25 to $1.5 \mathrm{eV}$ (Figure S4a). Such a slightly smaller variation in the $b c c$-type anion framework may be due to the more distorted lithium-anion tetrahedrons (Figure S10) leading to a more frustrated energy landscape for lithium ion migration ${ }^{34}$. The maximum variation of $E_{a}$ with respect to different anion charges at any constant lattice volume is $\sim 0.09 \mathrm{eV}$ for the oxygen- and sulfur-anion framework (Figure S8 and S9), indicating that $\mathrm{E}_{\mathrm{a}}$ of lithium ion migration along the Tet-Tet pathways in lithium compounds with the hcp-type anion frameworks are much less sensitive to the anion charge, which is similar to the case of lithium compounds with the bcc-type anion frameworks.

However, different from the $b c c$-type framework, $\mathrm{E}_{\mathrm{a}}$ variations with respect to different anion charges at any constant lattice volume in the hcp-type anion framework are not monotonically increasing or decreasing (Figure S8 and S9), while $\mathrm{E}_{\mathrm{a}}$ variations are monotonic for the Tet-Tet migration pathways in the $b c c$-type anion framework (Figure S5 and S6). It may be because the different local environments of lithium tetrahedral sites and the diverse triangular anion bottlenecks (transition states) in the $b c c$ - and hcp-type anion framework, as shown in Figure S10. Taking the hcp-type sulfur anion framework as an example (Figure 3a and S9), the sulfur anion charges, ranging from $-0.8 e$ to $-1.3 e$, can significantly decrease $\mathrm{E}_{\mathrm{a}}$, while $\mathrm{E}_{\mathrm{a}}$ gradually increase when the more negative sulfur anion charges in the range of $[-1.3 e,-1.8 e]$. It's worth noting that discussing $\mathrm{E}_{\mathrm{a}}$ variation with respect to the much more positive anion charges in the range of $[-0.5 e,-0.8 e]$ make no sense due to the very low probability to get such small anion charges in lithium sulfides (Figure S1). Moreover, it is found that the large lattice volumes can make low $\mathrm{E}_{\mathrm{a}}$ at any constant anion charge, and the effect of lattice volume on $E_{a}$ is larger than that of the anion charge for the Tet-Tet lithium ion migration. Thus, the moderate sulfur(oxygen) anion charges near $-1.3 e$ and especially large lattice volumes are beneficial to the fast lithium ion migration along the Tet-Tet migration pathways in lithium compounds with the hcp-type sulfur(oxygen) anion frameworks, although the effects of the Coulomb interaction depending on anion charge and lattice volume on the Tet-Tet lithium ion migration are limited. 
(a)

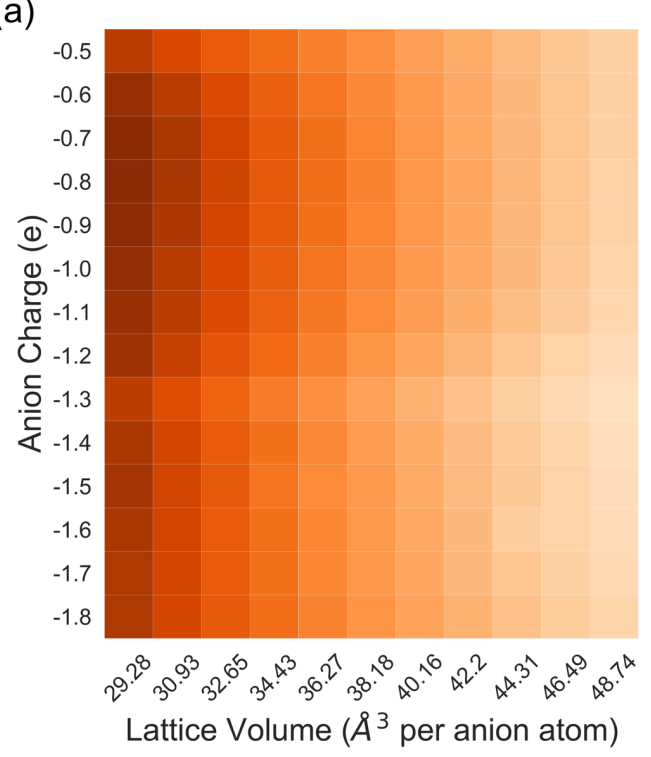

(c)

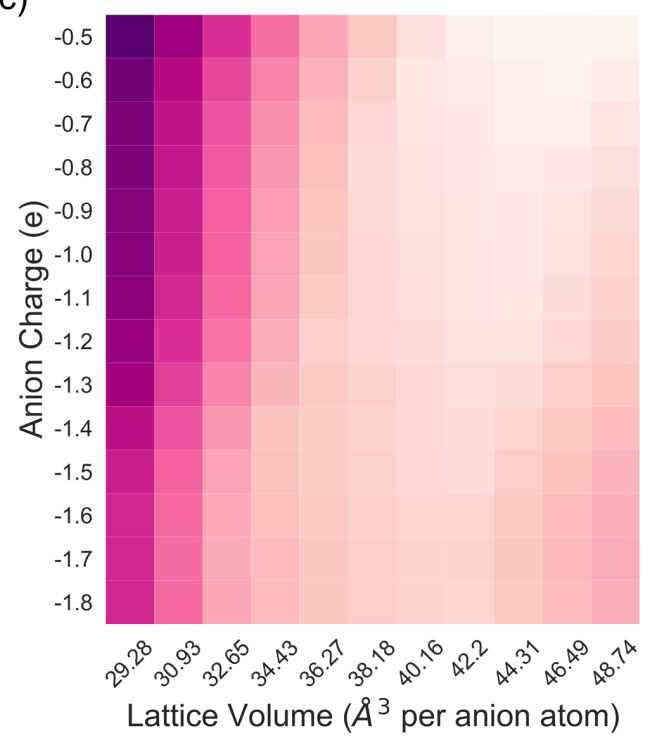

(b)
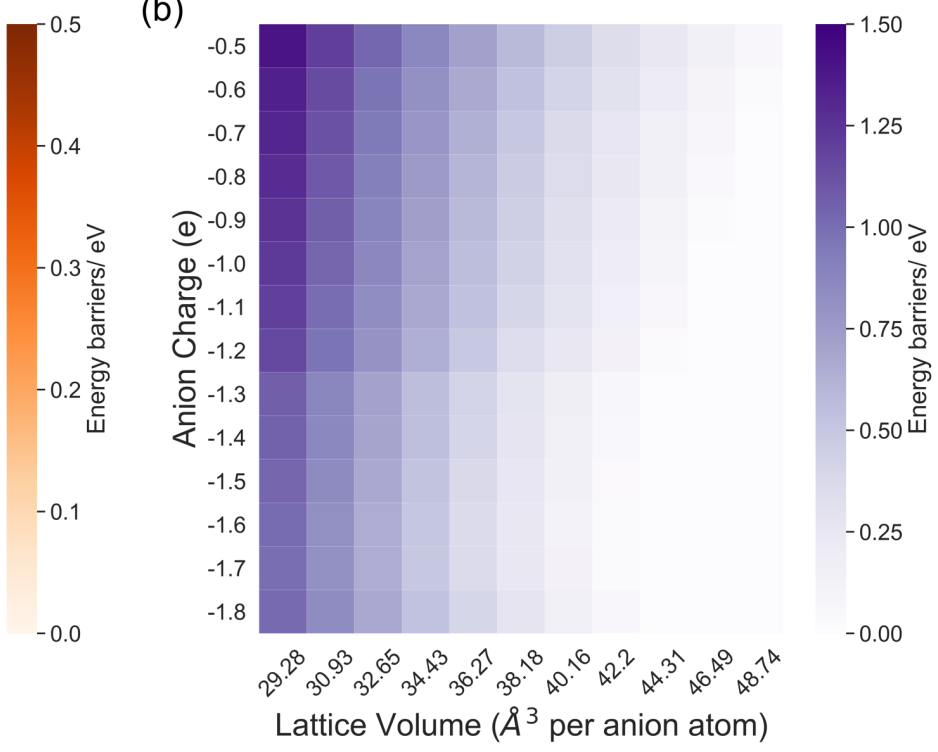

(d)

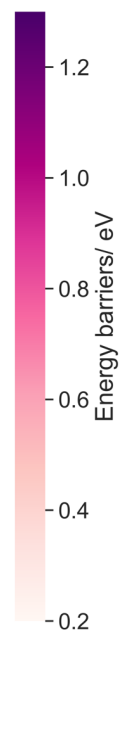

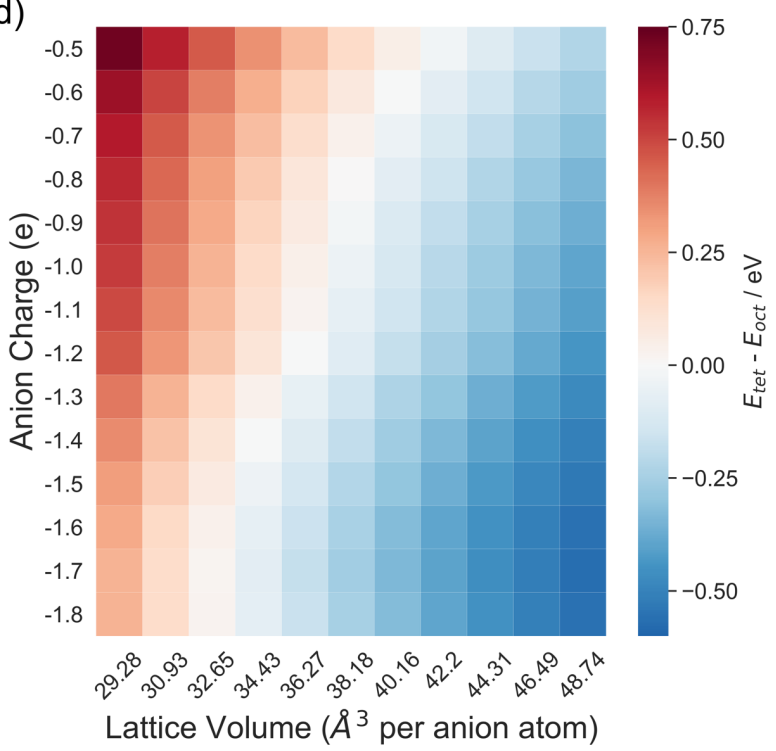

Figure 3. Heat maps of the calculated $\mathrm{E}_{\mathrm{a}}$ of lithium ion migration in the hcp-type sulfur anion frameworks along the (a) Tet-Tet, (b) Oct-Oct migration pathways with respect to anion charge and lattice volume; (c) the calculated $\mathrm{E}_{\mathrm{a}}$ of lithium ion migration between the two adjacent $O c t$ and Tet sites, and (d) the energy differences of the occupied lithium between the Tet site and Oct site in the $h c p$-type sulfur anion frameworks with respect to different anion charges and lattice volumes.

\subsubsection{Oct-Oct pathway}

And then we focus on another direct lithium ion migration pathway in the hcp-type anion frameworks, namely Oct-Oct pathway. The calculated $\mathrm{E}_{\mathrm{a}}$ of lithium ion migration in the hcp-type sulfur(oxygen) anion frameworks along the Oct-Oct migration pathways with respect to anion charge and lattice volume are shown in Figure $3 \mathrm{~b}, \mathrm{~S} 11, \mathrm{~S} 12$ and $\mathrm{S} 13$. It can be found that $\mathrm{E}_{\mathrm{a}}$ 
variation ranging from 0 to $1.5 \mathrm{eV}$ in the whole anion charge and lattice volume space for the Oct-Oct migration pathways in the hcp-type sulfur(oxygen) anion framework is much wider than 0 to $0.5 \mathrm{eV}$ of the Tet-Tet migration pathways in the $b c c$ - and $h c p$-type sulfur(oxygen) anion frameworks. The maximum variation of $E_{a}$ with respect to different anion charges at any constant lattice volume is $\sim 0.4 \mathrm{eV}$ for the oxygen and sulfur anion framework (Figure S12 and S13), demonstrating that $\mathrm{E}_{\mathrm{a}}$ of lithium ion migration along the Oct-Oct pathways in lithium compounds with the $h c p$-type anion frameworks are sensitive to the variations of anion charge, and much different from the anion charge immunized Tet-Tet migration pathways. Interestingly, $\mathrm{E}_{\mathrm{a}}$ variations with respect to anion charge and lattice volume are monotonic. In the whole anion charge and lattice volume space, the more negative anion charges can reduce $\mathrm{E}_{\mathrm{a}}$ of lithium ion migration in the hcp-type anion framework along the Oct-Oct pathways at any constant lattice volume (Figure S12 and S13), and the large lattice volumes can make low $E_{a}$ at any constant anion charge. Over all, the Coulomb interactions depending on anion charge and lattice volume have great influences on $E_{a}$ of lithium ion migration along the Oct-Oct pathways in the hcp-type anion frameworks, and the more negative anion charges and large lattice volumes (lithium-anion bond lengths) are essential for achieving fast lithium ion migration along the Oct-Oct pathways in lithium compounds with the $h c p$-type anion frameworks.

\subsubsection{Oct-Tet pathway}

Lastly, we discuss the Oct-Tet pathway for lithium ion migration in the hcp-type anion frameworks, and it is the half of the Tet-Oct-Tet or Oct-Tet-Oct pathways, which are the most common lithium ion migration pathways in those lithium sulfides with the $h c p$-type sulfur anion frameworks ${ }^{35-37}$. The calculated $\mathrm{E}_{\mathrm{a}}$ of lithium ion migration along the Oct-Tet migration pathways, and the energy differences of lithium occupation between the Tet and Oct site ( $\mathrm{E}_{\text {tet-oct}}$ ) in the hcp-type sulfur(oxygen) anion frameworks with respect to anion charge and lattice volume are shown in Figure 3c, 3d, S14, S15 and S16. It can be observed that $E_{a}$ variation ranging from 0.2 to $1.4 \mathrm{eV}$ and Etet-oct variation from -0.76 to $0.9 \mathrm{eV}$ in the whole anion charge and lattice volume space for the Oct-Tet migration pathways in the hcp-type sulfur(oxygen) anion frameworks are very wide, indicating the anion charge and lattice volume have significant effects on $E_{a}$ and $E_{\text {tet-oct values for }}$ both the oxygen and sulfur anion systems. Within different lattice volume regions, the anion charges have different effects on $\mathrm{E}_{\mathrm{a}}$ for lithium ion migration along the Oct-Tet migration pathways in the $h c p$-type anion frameworks. For any specific lattice volume, the more negative anion charges 
consistently reduce $E_{\text {tet-oct }}$ values (Figure $3 \mathrm{~d}$ and S14b), and stabilizing lithium-anion tetrahedra. At any constant anion charge, $\mathrm{E}_{\text {tet-oct }}$ values vary from positive to negative, and the relative stabilities of lithium-anion tetrahedra gradually increase when lattice volumes get larger. In sum, both the much negative anion charge and large lattice volume make lithium prefer to occupy the Tet sites.

Facetgrid plots in Figure S15 and S16 show the anion charge dependent $\mathrm{E}_{\mathrm{a}}$ at different lattice volumes. Taking the sulfur anion framework as an example, when the sulfur anion lattices volume are small, e.g. with a value of $\sim 32.65 \AA^{3} /$ atom, the relative energies of $\mathrm{LiS}_{4}$ are all higher than those of $\mathrm{LiS}_{6}\left(\mathrm{E}_{\text {tet-oct }}>0\right.$, Figure 3d), indicating lithium is most stable at the Oct site and prefers to have the octahedral lithium occupation pattern with respect to small sulfur anion lattice volume, which is consistent with those $h c p$-type lithium sulfides with relative small lattice volumes ( $<39 \AA^{3} /$ atom) exhibiting octahedral lithium coordination environments (Table S1). Additionally, the more negative sulfur anion charges would lower the relative energies of $\mathrm{LiS}_{4}$, and hence reduce the corresponding $\mathrm{E}_{\mathrm{a}}$ for lithium ion migration along the Oct-Tet-Oct migration pathways in the hcp-type sulfur anion frameworks with smaller lattice volumes (Figure $3 \mathrm{~d}$ and S16). For the sulfur anion sublattice systems with medium lattice volumes, e.g. $\sim 36.27 \AA^{3} /$ atom, the relative energies of $\mathrm{LiS}_{4}$ are higher than those of $\mathrm{LiS}_{6}\left(\mathrm{E}_{\text {tet-oct }}>0\right.$, Figure $\left.3 \mathrm{~d}\right)$ for the systems with the less negative sulfur anion charges $\left(q_{\mathrm{S}} \in[-0.5 e,-1.1 e]\right)$. While the more negative sulfur anion charges $\left(q_{\mathrm{S}} \in\right.$ $[-1.1 e,-1.8 e])$ make the relative energies of $\mathrm{LiS}_{4}$ lower than those of $\mathrm{LiS}_{6}\left(\mathrm{E}_{\text {tet-oct }}<0\right)$, and thereby the Oct sites no longer keep stable. Thereby, with the negative sulfur anion charges increasing from -0.5 to $-1.8 e, \mathrm{E}_{\mathrm{a}}$ first decrease and then increase (Figure S16). At larger lattice volumes, e.g. 40.16 $\AA^{3} /$ atom, the relative energies of $\mathrm{LiS}_{4}$ are lower than those of $\mathrm{LiS}_{6}\left(\mathrm{E}_{\text {tet-oct }}<0\right.$, Figure $\left.3 \mathrm{~d}\right)$, indicating lithium prefers the Tet site at the large lattice volume, as shown in Table S1 that the hcp-type lithium sulfides with large sulfur lattice volumes ( $>39 \AA^{3} /$ atom) showing the tetrahedral lithium occupation patterns. In short, the much negative anion charges would deliver high $\mathrm{E}_{\mathrm{a}}$ for the tetrahedral lithium ion migration along the Tet-Oct-Tet pathways in the hcp-type anion frameworks, but lower $\mathrm{E}_{\mathrm{a}}$ for the octahedral lithium ion migration along the Oct-Tet-Oct pathways.

For studying lithium ion migration in real lithium compounds with the $h c p$-type anion frameworks with respect to anion charge and lattice volume, we should first figure out the stable lithium occupation site and main lithium ion migration path. Table S1 and S2 clearly show that the lithium coordination environment in the most lithium sulfides with the $h c p$-type anion frameworks is tetrahedrally coordinated, and thereby the main lithium ion migration pathways in lithium sulfides are Tet-Tet and Tet-Oct-Tet. Because $\mathrm{E}_{\mathrm{a}}$ of lithium ion migration along the Tet-Tet pathways in the 
$h c p$-type anion frameworks is much less sensitive to the anion charge and lattice volume than that of the Tet-Oct-Tet pathway, so the anion charge and lattice volume dependent $\mathrm{E}_{\mathrm{a}}$ of lithium ion migration in those lithium compounds with the hcp-type anion frameworks have the main pathway characteristic of Tet-Oct-Tet, and eventually the little negative anion charges would deliver low $\mathrm{E}_{\mathrm{a}}$ for the tetrahedral lithium ion migration in the $h c p$-type anion frameworks. Thus, the little negative anion charges and large lattice volumes (lithium-anion bond lengths) are necessary for achieving fast lithium ion migration in those lithium compounds with the $h c p$-type anion frameworks and stable tetrahedral lithium occupations.

The above analyses on the anion charge dependent $\mathrm{E}_{\mathrm{a}}$ for the hcp-type anion framework model are also validated by some real lithium sulfides. Figure 4 and Table S2 show some experimentally determined and theoretically calculated $E_{a}$ for the tetrahedral lithium ion migration in those lithium sulfides possessing $h c p$-type sulfur anion frameworks with respect to the sulfur anion Bader charges. As mentioned in section 3.1 (Figure S2), the Bader charges of lithium ion in different compounds don't change a lot, and thereby the anion charge variation of different lithium compounds can be regarded as resulting from the non-lithium cation element, which is fundamentally determined by the electronegativity difference between anion element and non-lithium cation element. In other words, the electronegativity of the non-lithium cation element $\mathrm{M}$ of Li-M-S compounds with $h c p$-type sulfur anion frameworks can significantly affect $\mathrm{E}_{\mathrm{a}}$ of lithium ion migration. It can be seen from Figure 4 that the non-lithium cation element $M$ has great impacts on $E_{a}$ for the tetrahedral lithium ion migration in those lithium sulfides with $h c p$-type sulfur anion frameworks. For example of the structurally similar $\mathrm{LiMS}_{2}(\mathrm{M}=\mathrm{In}$ and $\mathrm{Ga})$ with the same space group (Pna2 ${ }_{1}$, No.33), the Ga element with relative larger electronegativity donates less electrons to its adjacent sulfur anions, and makes the smaller average Bader charge of sulfur anions in $\mathrm{LiGaS}_{2}$ than that of $\mathrm{LiInS}_{2}$, eventually leading to a lower $E_{a}$ of the tetrahedral lithium ion migration. Moreover, the same trends are also observed in $\mathrm{Li}_{3} \mathrm{MS}_{3}(\mathrm{M}=\mathrm{Al}$ and $\mathrm{Sb}), \mathrm{Li}_{3} \mathrm{MS}_{4}(\mathrm{M}=\mathrm{P}, \mathrm{As}$, and $\mathrm{Sb})$, and $\mathrm{Li}_{4} \mathrm{MS}_{4}(\mathrm{M}=\mathrm{Ge}$ and $\mathrm{Sn})$. For the most lithium sulfides with $h c p$-type sulfur anion frameworks in Table S2, there is no linear positive relationship between lattice volume and Li-S bond length (Figure S17), which is quite different from the ideal anion framework model. For $\mathrm{Li}_{3} \mathrm{MS}_{4}(\mathrm{M}=\mathrm{P}, \mathrm{As}$, and $\mathrm{Sb})$ and $\mathrm{Li}_{4} \mathrm{MS}_{4}$ $(\mathrm{M}=\mathrm{Ge}$ and $\mathrm{Sn})$, their Li-S bond lengths are found to be negatively related to the corresponding lattice volumes, and the larger Li-S bond length of $\mathrm{Li}_{3} \mathrm{PS}_{4}$ further contribute to reduced $\mathrm{E}_{\mathrm{a}}$ together with the less negative charges of sulfur anion. But for $\operatorname{LiMS}_{2}(\mathrm{M}=\mathrm{In}$ and $\mathrm{Ga})$ and $\mathrm{Li}_{3} \mathrm{MS} \mathrm{S}_{3}(\mathrm{M}=\mathrm{Al}$ and $\mathrm{Sb}$ ), the larger $\mathrm{Li}-\mathrm{S}$ bond lengths don't make the total $\mathrm{E}_{\mathrm{a}}$ reduced as expected, while the increased $E_{a}$ are mirrors of the more negative sulfur anion charges, indicating the weight of anion 
charge contributing to the total $\mathrm{E}_{\mathrm{a}}$ of those sulfides is very high. And yet there is no denying that the large lattice volume (lithium-anion bond length) can lower $E_{a}$ to a certain extent when fixing the anion charges ${ }^{11,20}$. Above all, these $E_{a}$ variation tendencies with respect to the substituted non-lithium cation element $\mathrm{M}$ validate our anion charge-lattice volume maps of the hcp-type anion framework models.
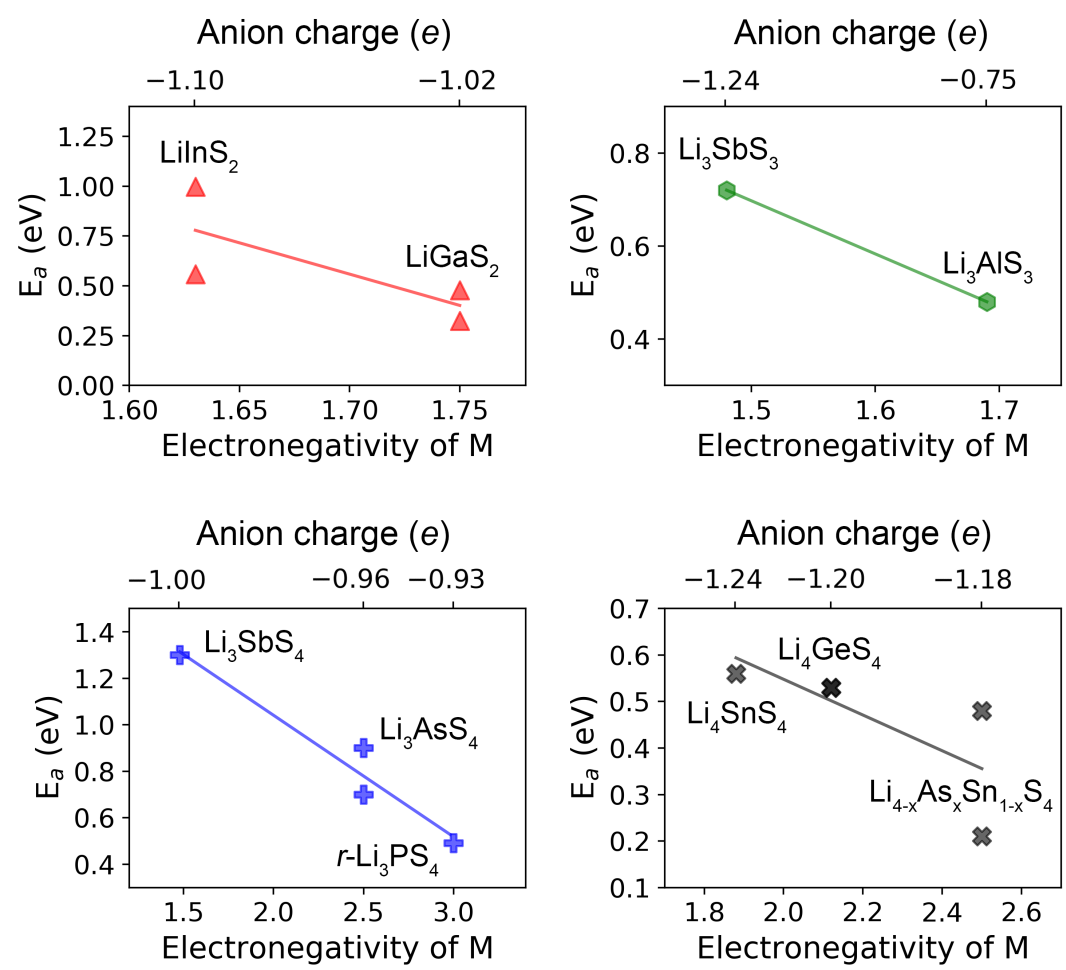

Figure 4. Experimentally determined and theoretically calculated activation energy barriers $E_{a}$ of the tetrahedral lithium ion migration in lithium sulfides possessing the hcp-type sulfur anion frameworks with respect to the electronegativity of non-lithium cation element $M$ and the sulfur anion Bader charges ${ }^{35-41}$. Lithium sulfides in each subplot are structurally similar with the same space group. The corresponding data of $\mathrm{E}_{\mathrm{a}}$, anion charge and electronegativity of $\mathrm{M}$ are also listed in Table S2.

\subsection{New rules for developing alkali-metal superionic conductors}

In lithium compounds, the anion charges are significantly influenced by the non-lithium metal elements $^{19,32}$. The atomic radius and valence electron configuration of the non-lithium metal element determines its coordination environment and lattice volume, eventually affecting the anion charge, lithium-anion bond length and lithium occupation pattern. Our model analyses of the $f c c-$, $b c c$ - and $h c p$-type anion frameworks clearly demonstrate that the anion charge and lattice volume (lithium-anion bond length) dramatically affect lithium ion migration especially for Oct-Tet and 
Oct-Oct pathways. Therefore, it makes sense to gain low $\mathrm{E}_{\mathrm{a}}$ for lithium ion migration by choosing the most suitable non-lithium metal element without changing the crystal structure framework a lot.

Based on the deep understandings of the anion framework models (Figure 2, 3 and S3), two general rules for developing new ternary $\mathrm{ABC}$ type lithium, sodium or even multivalent alkali-metal superionic conductors with $f c c$-, $b c c$ - and $h c p$-type anion frameworks are proposed here: (i) for the desired alkali-metal superionic conductors with alkali-metal (A) ion tetrahedral occupations, the small electronegativity difference between the anion elements $\mathrm{C}$ and the non-mobile cation elements $\mathrm{B}$ is necessary for obtaining fast alkali-metal (A) ion migration along the Tet-Tet and Tet-Oct-Tet pathways (Figure 5a), and the proper non-mobile cation elements B should give preference to those main group elements located at the right top of the periodic table of elements with large electronegativities, which are close to but less than that of $\mathrm{C}$ elements, as shown in Figure $5 \mathrm{~b}$. The chemical components of the most sulfide-type superionic conductors with tetrahedral lithium ion migration along the Tet-Tet and Tet-Oct-Tet pathways, such as $\mathrm{Li}_{10} \mathrm{GeP}_{2} \mathrm{~S}_{12}{ }^{9}, \mathrm{Li}_{3} \mathrm{PS}_{4}{ }^{42-43}, \mathrm{Li}_{1+2 \times} \mathrm{Zn}_{1-\mathrm{x}} \mathrm{PS}_{4}{ }^{14}$ and $\mathrm{Li}_{2} \mathrm{CuPS}_{4}{ }^{24}$, are completely in conformity with this tetrahedron rule; (ii) for the desired alkali-metal superionic conductors with alkali-metal (A) ion octahedral occupations, the large electronegativity difference between the anion elements $\mathrm{C}$ and the non-mobile cation elements $\mathrm{B}$ is essential for getting fast alkali-metal (A) ion migration along the Oct-Oct and Oct-Tet-Oct pathways (Figure 5a), and the promising non-mobile cation elements B should give preference to those subgroup transition-metal elements located at the left bottom of the periodic table with small electronegativities, as shown in Figure 5b. The chemical components of the recent reported halide-type lithium superionic conductors, e.g. $\mathrm{Li}_{3} \mathrm{YCl}_{6}{ }^{22}, \mathrm{Li}_{3} \mathrm{YBr}_{6}{ }^{22}$ and $\mathrm{Li}_{3} \mathrm{LaI}_{6}{ }^{21}$ with octahedral lithium ion migration along the Oct-Tet-Oct pathways, fit this octahedron rule well, and their lithium ionic conductivities can be further enhanced by doping with the appropriate rare-earth metal elements, whose electronegativity values are less than yttrium element. 
(a)

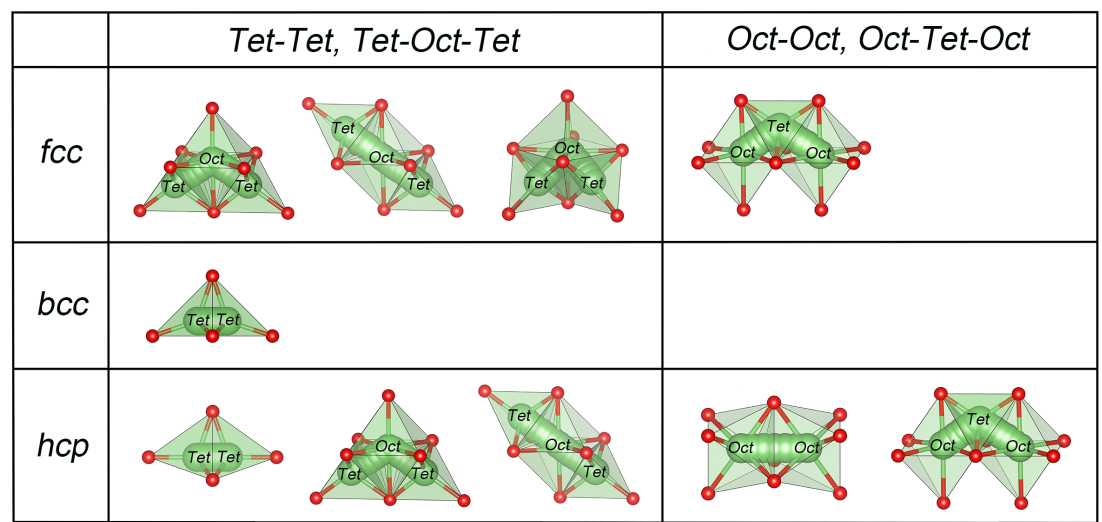

(b)

\begin{tabular}{|c|c|c|c|c|c|c|c|c|c|c|c|c|c|c|c|c|c|}
\hline H & & & & B & & super & ionic & $\mathrm{C}$ co & ndu & ictors & & & & & & & \\
\hline Li & $\mathrm{Be}$ & & & & & & & & & & & B & C & $\mathbf{N}$ & 0 & $F$ & \\
\hline $\mathrm{Na}$ & Mg & & & & & & & & & & & Al & Si & $\mathbf{P}$ & $s$ & $\mathrm{Cl}$ & \\
\hline K & $\mathrm{Ca}$ & Sc & $\mathrm{Ti}$ & V & $\mathrm{Cr}$ & $M n$ & $\mathrm{Fe}$ & Co & $\mathrm{Ni}$ & $\mathrm{Cu}$ & $\mathrm{Zn}$ & Ga & Ge & As & $\mathrm{Se}$ & $\mathrm{Br}$ & $\mathrm{Kr}$ \\
\hline & $\mathrm{Sr}$ & $\mathbf{Y}$ & $\mathrm{Zr}$ & Nb & Mo & Tc & Ru & $\mathbf{R h}$ & $\mathbf{P d}$ & $\mathrm{Ag}$ & $\mathrm{Cd}$ & In & Sn & $\mathrm{Sb}$ & $\mathrm{Te}$ & 1 & \\
\hline 1 & $\mathrm{Ba}$ & & $\mathrm{Hf}$ & $\mathrm{Ta}$ & $\mathbf{W}$ & $\operatorname{Re}$ & Os & Ir & $\mathrm{Pt}$ & $\mathrm{Au}$ & $\mathrm{Hg}$ & TI & $\mathrm{Pb}$ & $\mathrm{Bi}$ & Po & At & $\mathbf{R n}$ \\
\hline - & $\mathbf{R}$ & & Rf & $\mathrm{Db}$ & $\mathrm{Sg}$ & $\mathrm{Bh}$ & Hs & Mt & Ds & F & Cn & $\mathrm{Nh}$ & FI & Mc & Lv & Ts & \\
\hline
\end{tabular}

La Ce Pr Nd Pm Sm Eu Gd Tb Dy Ho Er Tm Yb Lu

Ac Th Pa U Np Pu Am Cm Bk Cf Es Fm Md No Lr
A Mobile alkali-metal cation elements
B Main group non-mobile cation elements
B Subgroup transition-metal non-mobile cation elements
C Anion elements

Figure 5. (a) Migration pathways of the tetrahedrally and octahedrally occupied alkali-metal (A) ion in the $f c c$-, $b c c$ - and $h c p$-type anion frameworks. Considering the different relative positions of the initial and final Tet sites, there are three and two kinds of Tet-Oct-Tet pathways for the $f c c$ and $h c p$ type anion frameworks, respectively; (b) the recommended choices of the non-mobile cation element B (olive and orange areas) in the periodic table of element for achieving fast alkali-metal (A) ion migration in the $\mathrm{ABC}$ ternary compounds. 


\section{Conclusions}

In this work, based on the density functional theory calculations and anion framework models, we have figured out the anion charge and lattice volume (lithium-anion bond length) dependent lithium ion diffusion in lithium compounds. The anion framework models clearly demonstrate that the anion charge and lattice volume dramatically affect $E_{a}$ for lithium ion migration, which is validated by some reported lithium sulfides. For the tetrahedrally occupied lithium, the less negative anion charge is, the lower the lithium ion migration barrier is. While for the octahedrally occupied lithium, the more negative anion charge is, the lower the lithium ion migration barrier is. The large lattice volume (lithium-anion bond length) can lower $\mathrm{E}_{\mathrm{a}}$ to a certain extent. Lithium ion direct migrations along the direct Tet-Tet pathway in the bcc-or hcp-type anion framework are less sensitive to anion charge and lattice volume than other pathways. Moreover, new design rules for developing advanced alkali-metal superionic conductors were proposed based on the full understandings of anion framework model. Choosing the most suitable non-mobile cation element without changing the crystal structure framework to get the desired electronegativity difference between the anion element and non-mobile cation element, can eventually achieve low $E_{a}$ for alkali-metal ion migration. For the desired alkali-metal superionic conductors with alkali-metal ion tetrahedral occupations, the small electronegativity difference between the anion element and four-coordinated non-mobile cation element is necessary for obtaining fast alkali-metal ion migration along the Tet-Tet and Tet-Oct-Tet pathways, and the fine non-mobile cation element should give preference to those elements located at the right top of the periodic table of elements with large electronegativity. For the alkali-metal superionic conductors with alkali-metal ion octahedral occupations, the large electronegativity difference between the anion element and six-coordinated non-mobile cation element is essential for getting fast alkali-metal ion migration along the Oct-Oct and Oct-Tet-Oct pathways, and the fine non-mobile cation element should give preference to the elements located at the left bottom of the periodic table with small electronegativity.

\section{Acknowledgements}

This work is supported by the National Natural Science Foundation of China (51602196), Shanghai Automotive Industry Corporation (1714), and Materials Genome Initiative Center at Shanghai Jiao Tong University. Z. Xu is much grateful to the support of the China Scholarship Council (CSC, 
scholarship No. 201906230117). All simulations were performed at the Shanghai Jiao Tong University High Performance Computing Center.

\section{Supporting information}

Scatter distributions of anion Bader charge and lattice volume of lithium compounds; Histogram distributions of Bader charges of lithium ion in compounds; Heat maps and facetgrid plots of calculated $\mathrm{E}_{\mathrm{a}}$ for oxygen and sulfur anion frameworks; Matched $b c c$ and $h c p$ anion frameworks for lithium sulfides from the MP database; Experimentally determined and theoretically calculated $\mathrm{E}_{\mathrm{a}}$ of tetrahedral Li ion migration in lithium sulfides with $h c p$-type sulfur anion frameworks; Scatter distributions of lattice volumes and average Li-S bond lengths of lithium sulfides with tetrahedral lithium occupations and $h c p$-type sulfur anion frameworks.

\section{Author's information}

Corresponding Authors

*E-mail: hong.zhu@sjtu.edu.cn

Notes

The authors declare no competing financial interest.

\section{References}

1. Pomerantseva, E.; Bonaccorso, F.; Feng, X.; Cui, Y.; Gogotsi, Y., Energy storage: The future enabled by nanomaterials. Science 2019, 366, 969.

2. Dunn, B.; Kamath, H.; Tarascon, J. M., Electrical energy storage for the grid: a battery of choices. Science 2011, 334, 928-935.

3. Harper, G.; Sommerville, R.; Kendrick, E.; Driscoll, L.; Slater, P.; Stolkin, R.; Walton, A.; Christensen, P.; Heidrich, O.; Lambert, S.; Abbott, A.; Ryder, K.; Gaines, L.; Anderson, P., Recycling lithium-ion batteries from electric vehicles. Nature 2019, 575 (7781), 75-86. 
4. Zhang, Z.; Shao, Y.; Lotsch, B.; Hu, Y.-S.; Li, H.; Janek, J.; Nazar, L. F.; Nan, C.-W.; Maier, J.; Armand, M.; Chen, L., New horizons for inorganic solid state ion conductors. Energy \& Environmental Science 2018, 8, 1945-1976.

5. Xabier Jude, G. G. E., Chunmei Li, Lide M. Rodriguez-Martinez,; Heng Zhang, a. M. A., Opportunities for Rechargeable Solid-State Batteries Based on Li-Intercalation Cathodes. Joule 2018, $2,1-17$.

6. Sun, C.; Liu, J.; Gong, Y.; Wilkinson, D. P.; Zhang, J., Recent advances in all-solid-state rechargeable lithium batteries. Nano Energy 2017, 33, 363-386.

7. Murugan, R.; Thangadurai, V.; Weppner, W., Fast Lithium Ion Conduction in Garnet-Type $\mathrm{Li}_{7} \mathrm{La}_{3} \mathrm{Zr}_{2} \mathrm{O}_{12}$. Angew. Chem. Int. Ed. 2007, 46 (41), 7778-7781.

8. Morimoto, H.; Awano, H.; Terashima, J.; Shindo, Y.; Nakanishi, S.; Ito, N.; Ishikawa, K.; Tobishima, S.-i., Preparation of lithium ion conducting solid electrolyte of NASICON-type $\mathrm{Li}_{1+\mathrm{x}} \mathrm{Al}_{\mathrm{x}} \mathrm{Ti}_{2-\mathrm{x}}\left(\mathrm{PO}_{4}\right)_{3}(\mathrm{x}=0.3)$ obtained by using the mechanochemical method and its application as surface modification materials of $\mathrm{LiCoO}_{2}$ cathode for lithium cell. J. Power Sources 2013, 240, 636-643.

9. Kamaya, N.; Homma, K.; Yamakawa, Y.; Hirayama, M.; Kanno, R.; Yonemura, M.; Kamiyama, T.; Kato, Y.; Hama, S.; Kawamoto, K.; Mitsui, A., A lithium superionic conductor. Nature materials 2011, 10 (9), 682-686.

10. Seino, Y.; Ota, T.; Takada, K.; Hayashi, A.; Tatsumisago, M., A sulphide lithium super ion conductor is superior to liquid ion conductors for use in rechargeable batteries. Energy Environ. Sci. 2014, 7 (2), 627-631.

11. Wang, Y.; Richards, W. D.; Ong, S. P.; Miara, L. J.; Kim, J. C.; Mo, Y.; Ceder, G., Design principles for solid-state lithium superionic conductors. Nature materials 2015, 14, 1026.

12. Ong, S. P.; Mo, Y.; Richards, W. D.; Miara, L.; Lee, H. S.; Ceder, G., Phase stability, electrochemical stability and ionic conductivity of the $\mathrm{Li}_{10 \pm 1} \mathrm{MP}_{2} \mathrm{X}_{12}(\mathrm{M}=\mathrm{Ge}, \mathrm{Si}, \mathrm{Sn}, \mathrm{Al}$ or $\mathrm{P}$, and $\mathrm{X}=\mathrm{O}, \mathrm{S}$ or Se) family of superionic conductors. Energy \& Environmental Science 2013, 6 (1), 148-156.

13. He, X.; Bai, Q.; Liu, Y.; Nolan, A. M.; Ling, C.; Mo, Y., Crystal Structural Framework of Lithium Super-Ionic Conductors. Advanced Energy Materials 2019, 9 (43), 1902078.

14. Richards, W. D.; Wang, Y.; Miara, L. J.; Kim, J. C.; Ceder, G., Design of $\mathrm{Li}_{1+2 \times} \mathrm{Zn}_{1-\mathrm{x}} \mathrm{PS}_{4}$, a new lithium ion conductor. Energy \& Environmental Science 2016, 9 (10), 3272-3278.

15. Kato, Y.; Hori, S.; Saito, T.; Suzuki, K.; Hirayama, M.; Mitsui, A.; Yonemura, M.; Iba, H.; Kanno, R., High-power all-solid-state batteries using sulfide superionic conductors. Nature Energy 2016, 1 (4), 16030. 
16. Adeli, P.; Bazak, J. D.; Park, K. H.; Kochetkov, I.; Huq, A.; Goward, G. R.; Nazar, L. F., Boosting Solid-State Diffusivity and Conductivity in Lithium Superionic Argyrodites by Halide Substitution. Angew. Chem. Int. Ed. Engl. 2019, 58 (26), 8681-8686.

17. Deiseroth, H. J.; Kong, S. T.; Eckert, H.; Vannahme, J.; Reiner, C.; Zaiss, T.; Schlosser, M., $\mathrm{Li}_{6} \mathrm{PS}_{5} \mathrm{X}$ : a class of crystalline Li-rich solids with an unusually high $\mathrm{Li}^{+}$mobility. Angew. Chem. Int Ed. Engl. 2008, 47 (4), 755-8.

18. Xu, B.; Meng, S., Factors affecting Li mobility in spinel $\mathrm{LiMn}_{2} \mathrm{O}_{4}-\mathrm{A}$ first-principles study by GGA and GGA+U methods. J. Power Sources 2010, 195 (15), 4971-4976.

19. Xu, Z. M.; Bo, S. H.; Zhu, H., $\mathrm{LiCrS}_{2}$ and $\mathrm{LiMnS}_{2}$ Cathodes with Extraordinary Mixed Electron-Ion Conductivities and Favorable Interfacial Compatibilities with Sulfide Electrolyte. ACS applied materials \& interfaces 2018, 10, 36941-36953.

20. Wang, S.; Bai, Q.; Nolan, A. M.; Liu, Y.; Gong, S.; Sun, Q.; Mo, Y., Lithium Chlorides and Bromides as Promising Solid-State Chemistries for Fast Ion Conductors with Good Electrochemical Stability. Angew. Chem. Int. Ed. 2019, 58 (24), 8039-8043.

21. Xu, Z.; Chen, X.; Liu, K.; Chen, R.; Zeng, X.; Zhu, H., Influence of Anion Charge on Li Ion Diffusion in a New Solid-State Electrolyte, $\mathrm{Li}_{3} \mathrm{LaI}_{6}$. Chem. Mater. 2019, 31 (18), 7425-7433.

22. Asano, T.; Sakai, A.; Ouchi, S.; Sakaida, M.; Miyazaki, A.; Hasegawa, S., Solid Halide Electrolytes with High Lithium-Ion Conductivity for Application in $4 \mathrm{~V}$ Class Bulk-Type All-Solid-State Batteries. Adv. Mater. 2018, 30, 1803075.

23. Xu, Z.; Chen, X.; Chen, R.; Li, X.; Zhu, H., Anion charge-lattice volume dependent Li ion migration in compounds with the face-centered cubic anion frameworks. arXiv preprint arXiv:1910.11545 2019.

24. Xu, Z.; Chen, R.; Zhu, H., $\mathrm{A} \mathrm{Li}_{2} \mathrm{CuPS}_{4}$ superionic conductor: a new sulfide-based solid-state electrolyte. Journal of Materials Chemistry A 2019, 7 (20), 12645-12653.

25. Blöchl, P. E., Projector augmented-wave method. Physical Review B 1994, 50 (24), 17953-17979.

26. Kohn, W.; Sham, L. J., Self-Consistent Equations Including Exchange and Correlation Effects. Phys. Rev. 1965, 140 (4A), A1133-A1138.

27. Perdew, J. P.; Burke, K.; Ernzerhof, M., Generalized Gradient Approximation Made Simple. Phys. Rev. Lett. 1996, 77 (18), 3865-3868.

28. Monkhorst, H. J.; Pack, J. D., Special points for Brillouin-zone integrations. Physical Review B 1976, 13 (12), 5188-5192. 
29. Henkelman, G.; Jónsson, H., Improved tangent estimate in the nudged elastic band method for finding minimum energy paths and saddle points. The Journal of Chemical Physics 2000, 113 (22), 9978-9985.

30. Curtarolo, S.; Setyawan, W.; Wang, S.; Xue, J.; Yang, K.; Taylor, R. H.; Nelson, L. J.; Hart, G. L. W.; Sanvito, S.; Buongiorno-Nardelli, M.; Mingo, N.; Levy, O., AFLOWLIB.ORG: A distributed materials properties repository from high-throughput ab initio calculations. Computational Materials Science 2012, 58, 227-235.

31. Li, K.; Xue, D., Estimation of electronegativity values of elements in different valence states. $J$. Phys. Chem. A 2006, 110 (39), 11332-7.

32. Krauskopf, T.; Culver, S. P.; Zeier, W. G., Bottleneck of Diffusion and Inductive Effects in $\mathrm{Li}_{10} \mathrm{Ge}_{1-\mathrm{x}} \mathrm{Sn}_{\mathrm{x}} \mathrm{P}_{2} \mathrm{~S}_{12}$. Chem. Mater. 2018, 30 (5), 1791-1798.

33. Liu, H.; Yang, Z.; Wang, Q.; Wang, X.; Shi, X., Atomistic insights into the screening and role of oxygen in enhancing the $\mathrm{Li}(+)$ conductivity of $\mathrm{Li}_{7} \mathrm{P}_{3} \mathrm{~S}_{11-\mathrm{x}} \mathrm{O}_{\mathrm{x}}$ solid-state electrolytes. Phys. Chem. Chem. Phys. 2019, 21 (48), 26358-26367.

34. Di Stefano, D.; Miglio, A.; Robeyns, K.; Filinchuk, Y.; Lechartier, M.; Senyshyn, A.; Ishida, H.; Spannenberger, S.; Prutsch, D.; Lunghammer, S.; Rettenwander, D.; Wilkening, M.; Roling, B.; Kato, Y.; Hautier, G., Superionic Diffusion through Frustrated Energy Landscape. Chem 2019, 5, 1-11.

35. Murayama, M.; Sonoyama, N.; Yamada, A.; Kanno, R., Material design of new lithium ionic conductor, thio-LISICON, in the $\mathrm{Li}_{2} \mathrm{~S}-\mathrm{P}_{2} \mathrm{~S}_{5}$ system. Solid State Ionics 2004, 170 (3-4), 173-180.

36. Sahu, G.; Lin, Z.; Li, J.; Liu, Z.; Dudney, N.; Liang, C., Air-stable, high-conduction solid electrolytes of arsenic-substituted $\mathrm{Li}_{4} \mathrm{SnS}_{4}$. Energy Environ. Sci. 2014, 7 (3), 1053-1058.

37. Kanno, R., Synthesis of a new lithium ionic conductor, thio-LISICON-lithium germanium sulfide system. Solid State Ionics 2000, 130 (1-2), 97-104.

38. Rao, F.-Y.; Ning, F.-H.; Jiang, L.-W.; Zeng, X.-M.; Wu, M.-S.; Xu, B.; Ouyang, C.-Y., First principle study of $\operatorname{LiXS}_{2}(\mathrm{X}=\mathrm{Ga}, \mathrm{In})$ as cathode materials for $\mathrm{Li}$ ion batteries. Chinese Physics $B$ 2016, 25 (2), 028202.

39. Gamon, J.; Duff, B. B.; Dyer, M. S.; Collins, C.; Daniels, L. M.; Surta, T. W.; Sharp, P. M.; Gaultois, M. W.; Blanc, F.; Claridge, J. B.; Rosseinsky, M. J., Computationally Guided Discovery of the Sulfide $\mathrm{Li}_{3} \mathrm{AlS}_{3}$ in the Li-Al-S Phase Field: Structure and Lithium Conductivity. Chem. Mater. 2019, 31 (23), 9699-9714.

40. Al-Qawasmeh, A.; Holzwarth, N. A. W., Computational Study of Li Ion Electrolytes Composed of $\mathrm{Li}_{3} \mathrm{AsS}_{4}$ Alloyed with $\mathrm{Li}_{4} \mathrm{GeS}_{4}$. J. Electrochem. Soc. 2016, 163 (9), A2079-A2088. 
41. Kwak, H.; Park, K. H.; Han, D.; Nam, K.-W.; Kim, H.; Jung, Y. S., Li ${ }^{+}$conduction in air-stable Sb-Substituted $\mathrm{Li}_{4} \mathrm{SnS}_{4}$ for all-solid-state Li-Ion batteries. J. Power Sources 2020, 446.

42. Tachez, M.; Malugani, J.-P.; Mercier, R.; Robert, G., Ionic conductivity of and phase transition in lithium thiophosphate $\mathrm{Li}_{3} \mathrm{PS}_{4}$. Solid State Ionics 1984, 14 (3), 181-185.

43. Homma, K.; Yonemura, M.; Kobayashi, T.; Nagao, M.; Hirayama, M.; Kanno, R., Crystal structure and phase transitions of the lithium ionic conductor $\mathrm{Li}_{3} \mathrm{PS}_{4}$. Solid State Ionics 2011, 182 (1), 53-58. 